\title{
High-Density Lipoprotein Mimetics: a Therapeutic Tool for Atherosclerotic Diseases
}

\author{
Masahiro Ikenaga ${ }^{1,2}$, Yasuki Higaki ${ }^{1,2}$, Keijiro Saku³ and Yoshinari Uehara ${ }^{1,2,3,4}$ \\ ${ }^{1}$ Faculty of Sports and Health Science, Fukuoka University, Fukuoka, Japan \\ ${ }^{2}$ The Fukuoka University Institute for Physical Activity, Fukuoka University, Fukuoka, Japan \\ ${ }^{3}$ Department of Cardiology, Fukuoka University School of Medicine, Fukuoka, Japan \\ ${ }^{4}$ Center for Preventive, Anti-aging and Regenerative Medicine, Fukuoka University Hospital, Fukuoka, Japan
}

\begin{abstract}
Clinical trials and epidemiological studies have revealed a negative correlation between serum highdensity lipoprotein (HDL) cholesterol levels and the risk of cardiovascular events. Currently, statin treatment is the standard therapy for cardiovascular diseases, reducing plasma low-density lipoprotein (LDL) cholesterol levels. However, more than half of the patients have not been able to receive the beneficial effects of this treatment.

The reverse cholesterol transport pathway has several potential anti-atherogenic properties. An important approach to HDL-targeted therapy is the optimization of HDL cholesterol levels and function in the blood to enhance the removal of circulating cholesterol and to prevent or mitigate inflammation that causes atherosclerosis. Cholesteryl ester transfer protein inhibitors increase HDL cholesterol levels in humans, but whether they reduce the risk of atherosclerotic diseases is unknown. HDL therapies using HDL mimetics, including reconstituted HDL, apolipoprotein (Apo) A-Imilano, ApoA-I mimetic peptides, or full-length ApoA-I, are highly effective in animal models. In particular, the Fukuoka University ApoA-I-mimetic peptide (FAMP) effectively removes cholesterol via the $\mathrm{ABCA} 1$ transporter and acts as an anti-atherosclerotic agent by enhancing the biological functions of HDL without elevating HDL cholesterol levels.

Our literature review suggests that HDL mimetics have significant atheroprotective potential and are a therapeutic tool for atherosclerotic diseases.
\end{abstract}

J Atheroscler Thromb, 2016; 23: 385-394.

Key words: Apolipoprotein A-I, Mimetic peptide, High-density lipoprotein, Atherosclerosis, Reverse cholesterol transport

\section{Introduction}

The main function of high-density lipoprotein (HDL) is to remove excess cholesterol in the peripheral tissues and organs. HDL directly or indirectly transports cholesterol to the liver, through apolipoprotein (Apo) B-containing lipoprotein with cholesterol ester transfer protein (CETP). In addition, HDL has anti-inflammatory, anti-oxidative, and anti-apoptotic

Address for correspondence: Yoshinari Uehara, Faculty of Sports and Health Science, Fukuoka University, Japan 8-19-1

Nanakuma, Jonan-ku, Fukuoka 814-0180 Japan

E-mail: ueharay@fukuoka-u.ac.jp

Received: October 25, 2015

Accepted for publication: November 24, 2015 functions and improves endothelium function, actions that are considered to be anti-atherogenic ${ }^{1)}$. (Fig. 1) The results of clinical trials and epidemiological studies support the hypothesis that HDL is "good cholesterol." Clinical trials and epidemiological studies have shown an inverse association between serum HDL cholesterol levels and the risk of cardiovascular events; however, recent studies have shown that a rise in HDL cholesterol is not necessarily associated with decreased risk of coronary artery disease $(\mathrm{CAD})^{2)}$. It is difficult to achieve anti-atherosclerotic success by HDL-increasing therapy because various factors involved in HDL metabolism, such as functional proteins, enzymes, or lipids, highly complicate its mechanism and such therapy must produce only anti-atherogenic HDL, but 


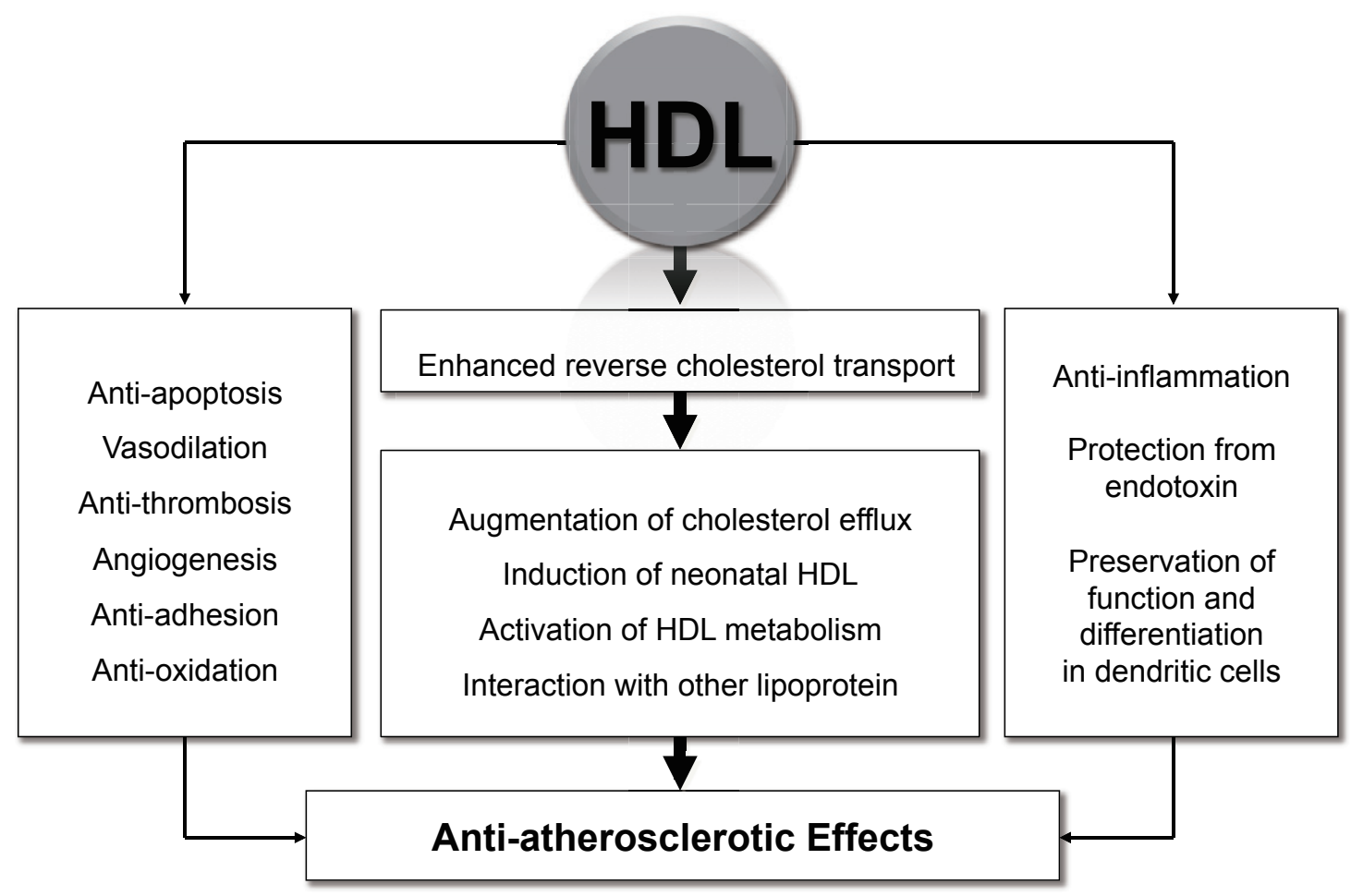

Fig. 1. Mechanisms by which HDL protects against atherosclerosis.

not atherogenic $\mathrm{HDL}^{3)}$.

Recently, several researchers have focused on a novel therapeutic strategy of atherosclerosis using HDL mimetics, such as reconstituted HDL (rHDL) and ApoA-I mimetic peptide, which are intended to directly increase HDL cholesterol levels or improve HDL function.

\section{HDL Targeted Therapy}

In 2012, the following two interesting clinical trials tested medication aimed at increasing HDL cholesterol levels: Atherothrombosis Intervention in Metabolic Syndrome with Low HDL/High Triglycerides and Impact on Global Health outcomes (AIMHIGH) and Efficacy and safety of dalcetrapib in patients with recent acute coronary syndrome (dalOUTCOMES); however, neither trial succeeded ${ }^{4,5)}$. The AIM-HIGH study used niacin, currently the strongest medicine available to increase serum HDL cholesterol, and medically lowered low-density lipoprotein (LDL) cholesterol levels were observed in highrisk patients, but there was no clinical benefit. The dal-OUTCOMES study was discontinued because of an absence of sufficient beneficial effects on cardiovascular disease, although the CETP inhibitor dalcetrapib increased serum HDL cholesterol levels ${ }^{5)}$. Whether CETP inhibitors reduced the risk of cardiovascular disease was not apparent. There are no positive results of clinical studies to date ${ }^{6,7)}$. An association between a gene mutation resulting in the elevation of HDL cholesterol and myocardial infarction was not confirmed; however, a mutation causing an increase in LDL cholesterol was shown to increase the risk of myocardial infarction ${ }^{8)}$. Therefore, it can be concluded that the pharmacotherapeutical induction of cholesterol concentration in HDL does not result in an increase in reverse cholesterol transport or reduction in the risk of future cardiovascular events.

CETP inhibitors that simply increase cholesterol levels in HDL particles by inactivation of cholesterol transference may be insufficient to reduce the risk of cardiovascular disease because HDL is composed of heterogeneous particles and its functions are defined by various factors, such as lipid metabolism and local inflammation ${ }^{1)}$. As a therapeutic approach to increasing HDL levels, various studies have focused both on increasing HDL cholesterol levels and on enhancing HDL function. HDL therapies that used injections of reconstituted HDL, ApoA-I mimetics, or full-length ApoA-I have been remarkably effective ${ }^{9,10)}$. Various animal studies have shown that a major protein of 
HDL, ApoA-I, suppressed the progression of atherosclerosis. An ApoA-I-overexpressing mouse model showed accelerated reverse cholesterol transport and suppressed atherosclerosis progression ${ }^{11,12)}$. In humans, the number of ApoA-I and HDL particles is a more important factor in cardiovascular disease than the HDL cholesterol level ${ }^{13,14)}$.

Low-HDL cholesterolemia is often observed as a characteristic component of metabolic syndrome, such as in people who are overweight or obese, those with glucose intolerance or obvious diabetes, those with hypertriglyceridemia, and those with high blood pressure, each of which contributes to atherosclerosis ${ }^{15)}$. However, exercise is considered to be a non-pharmacological treatment for dyslipidemia and is an important intervention for elevating serum HDL cholesterol level ${ }^{16)}$. Lifestyle modifications with regular exercise to increase daily physical activity and diet are well known initial steps for $\mathrm{CAD}$ prevention ${ }^{17)}$. In a randomized control trial investigating the effects of diet and exercise training, an improvement in HDL cholesterol levels was observed in an exercise training group after 12 weeks of intervention, but was not observed in a diet group $^{18)}$. Thus, exercise training can be an effective nonpharmacological treatment for increasing HDL cholesterol level.

Compared with increasing HDL cholesterol concentration, increasing the particle number of $\mathrm{HDL}$ and ApoA-I and the functions of HDL may contribute to reduce for the cardiovascular risk. One of the most effective strategies may be treatment with $\mathrm{HDL}$ mimetics, such as rHDL, ApoA-I, and ApoA-I mimetic peptide. ApoA-I mimetic peptide is constructed of a lesser number of amino acids than the full-length human ApoA-I. ApoA-I mimetics comprise a small peptide with an amphiphilic alpha helix structure and perform the major function of cholesterol efflux from cells as well as human ApoA-I and HDL. Here we outline four typical approaches using HDL mimetics.

\section{HDL Mimetics}

\section{ApoA-I Milano: MDCO-216 (formerly ETC-216)}

In 1980, a family lineage with a mutation in ApoA-I was discovered in Limone sul Gandain (northern Italy), and the mutant ApoA-I was termed Apo A-I Milano (ApoA-Iм). Interestingly, those with ApoA-Im did not suffer from atherosclerosis and were long-lived despite complication with severe low-HDL cholesterolemia ${ }^{19,20)}$. Later, studies showed that the ApoA-Iм is a mutant form of human ApoA-I that is generated by a point mutation that replaces an arginine with a cysteine residue at position 173. Higher levels of cellular cholesterol efflux mediated by ApoA-Iм in cognate patients than in subjects carrying ApoA-I are explained by the formation of ApoA-Im dimers linked by disulfide bonds between $\mathrm{C} 173$ residues ${ }^{21,22)}$. This linkage is suggested to be a reason for the absence of atherosclerosis.

Recently, there have been further studies of the medical use of rHDL, ETC-216 (new code name, MDCO-216, produced by The Medicines Company (Parsippany, NJ, USA) constructed from a recombinant ApoA-Im combined with phospholipids. ApoEdeficient mice receiving recombinant ApoA-Im showed reduced plaque formation and macrophage content compared with a control group ${ }^{23)}$. Administration of ApoA-Im can suppress the progression of atherosclerosis and fatty streak, one of the initial atherosclerotic changes in a rabbit model with lipid-rich plaques. The research group evaluated atherosclerotic changes following infusion (five times ETC-216 at 5, 10, 20, 40, or $150 \mathrm{mg} / \mathrm{kg}$ dose every 4 days) after 90 days of highcholesterol diet using intravascular ultrasonography (IVUS) and MRI in rabbits that had undergone perivascular injury in both carotid arteries. Atherosclerotic plaque formation assessed by IVUS in controls rapidly progressed during the treatment period, but was suppressed in a group treated with a low dose of ETC216 and regressed in a high-dose group ${ }^{24)}$. In humans, five weekly intravenous injections of ETC-216 reduced plaque volume in coronary arteries evaluated by IVUS in patients with acute coronary syndrome $(\mathrm{ACS})^{10)}$. Although the number of trials has been less, in prospective clinical trials, ETC-216 showed a greater plaque regression effect than 18 months of high-dose statin treatment ${ }^{10,25)}$.

\section{CER-001}

CER-001 is a HDL mimetic composed of recombinant ApoA-I and two types of phospholipids that are designed to mimic the beneficial properties of nascent pre- $\beta$ HDL. In vitro, CER-001 operates by reducing oxidized LDL from macrophages. In an animal study, CER-001 induced cholesterol efflux from the vascular wall and reduced plaque formation. A single treatment of CER-001 $(10 \mathrm{mg} / \mathrm{kg})$ resulted in increased cholesterol in the liver and feces of mice because of mobilization of unesterified cholesterol in circulating blood ${ }^{26)}$. In LDL-receptor-deficient mice, a familial hypercholesterolemia mouse model, infusion of CER-001 resulted in $17 \%$ and $32 \%$ reductions in plaque size and $17 \%$ and $23 \%$ reductions in lipid content after 5 and 10 doses, respectively, administered every 2 days ${ }^{26)}$. In a study investigating its anti-inflammatory effect, CER-001 suppressed the secretion of 
cytokines IL-6, IL-8, and MCP-1 and the GM-CSF response of lipopolysaccharide (LPS) activation on human endothelial cell in vitro. In an in vivo model, there was a decrease of vascular cell adhesion molecule (VCAM-1) expression by $16 \%$ (five doses) and $22 \%$ (10 doses). Injection of CER-001 resulted in a significant change in cholesterol mobilization to HDL in human ${ }^{26)}$.

Considering previous studies, CHI-SQUARE (Can HDL Infusions Significantly Quicken Atherosclerosis REgression) was designed ${ }^{27)}$. This study investigated the effects of CER-001 on atherosclerosis by IVUS and quantitative coronary angiography in patients with ACS. The number of patients was higher than that in the above clinical trial using ApoA-I, and a double-blinded, randomized trial was conducted. Patients were randomized to receive six weekly infusions of placebo or three doses of CER-001. Contrary to expectation, changes in total atheroma volume were not significantly different between the placebo and each dose of CER-001 and there were no differences among these groups during major adverse cardiovascular events.

Compared with the previous animal experimental studies, CER-001 did not show the beneficial effects on coronary plaque regression. On the other hand, CHI-SQUARE showed a dose-dependent increasing mobilization of cholesterol in circulating blood and a $45 \%$ increase in plasma level of free cholesterol following infusion with CER-001. However, this beneficial effect was not reflected in plaque regression analyzed by IVUS, and an assessment using a biomarker other than free cholesterol may be required to determine comparative efficacy against atherosclerotic plaques. Moreover, the anti-inflammatory action of HDL may be more important for plaque regression than for the major HDL function, i.e., cholesterol removal from cells.

\section{ApoA-I Mimetic Peptide: APL180 (L-4F)}

The two types of clinical approaches with MDCO-216 and CER-001 described above have issues in the cost and their stability, considering that human active ApoA-I is a 243-amino acid high-molecularweight protein. Therefore, ApoA-I mimetic peptides with few amino acids is being developed. One such peptide, 4F, consists of only 18 amino acids and presents four phenylalanine residues on its hydrophobic face.

The 4F peptide not only functions in cholesterol efflux but also exerts anti-oxidative and anti-inflammatory effects. APL180 (L-4F) and D-4F are similar peptides of the family. D-4F is an optical isomer of
L-4F, and the orally administrable D-4F, because of its $\mathrm{D}$-amino acid composition, is resistant to decomposition in vivo. In animal experiments using both $\mathrm{D}-4 \mathrm{~F}$ and $\mathrm{L}-4 \mathrm{~F}, 4 \mathrm{~F}$ showed a suppressive effect on atherosclerosis progression; inhibitory action against endotoxin damage; induction of adiponectin release in obese mice; reduction of inflammatory cytokines, such as IL- $1 \beta$ and TNF- $\alpha$; induction of paraoxonase (PON) activity; and improvement of insulin resistance ${ }^{28)}$. Moreover, another interesting function is caused by an interaction with lipids of $4 \mathrm{~F}$, which has a greater affinity for lipids than native ApoA-I; therefore, $4 \mathrm{~F}$ may reduce the oxidized lipids ${ }^{28}$. Recently, treatment with $4 \mathrm{~F}$ reduces lipid oxidation and improves the condition of pulmonary hypertension by increasing microRNA193-3p (miR193) expression in rodent models of pulmonary hypertension ${ }^{29)}$.

After incubation of LDL with human vascular endothelial cells, leukocyte migration is accelerated when the supernatant from the cells is reacted with leucocytes and promotion of migration is inhibited by HDL; however, the inhibitory effects differ from functional to dysfunctional HDL. Furthermore, it is possible to reveal the HDL function that is evaluated an inhibitory effect of the leukocyte migration as the HDL inflammatory index ${ }^{30)}$.

Following treatment with $4 \mathrm{~F}$ in a mouse model, dysfunctional HDL changes to functional HDL, i.e., the HDL inflammatory index is improved. In the first study using D-4F in humans, the HDL inflammatory index was significantly improved $4 \mathrm{~h}$ after a $300-\mathrm{mg}$ dose by oral administration and $2 \mathrm{~h}$ after a 500-mg dose compared with placebo, although plasma lipid and lipoprotein levels were unchanged ${ }^{31)}$. In an animal experiment, $\mathrm{L}-4 \mathrm{~F}$ and $\mathrm{D}-4 \mathrm{~F}$ showed similar efficacy, and $\mathrm{D}-4 \mathrm{~F}$ was found to remain for a long period in the liver and kidney without degradation; therefore, APL180 (L-4F) was used in subsequent clinical studies.

A clinical trial of APL180 in humans was reported in $2011^{32}$. In this study, L-4F was administered daily by either intravenous infusion for 8 days or subcutaneous injection for 28 days in patients with CAD. However, despite its previously published efficacy in animal models, treatment with L-4F did not improve HDL inflammatory index or PON activity. One of the issues identified in this study was that the dose of the peptides was lower than that in the initial clinical trial. A recent study found that a high dose of $4 \mathrm{~F}$ peptide was required for efficacy in the intestine.

\section{Apo A-I Mimetic Peptide: FAMP}

Fukuoka University Apo-A-I Mimetic Peptide 
a

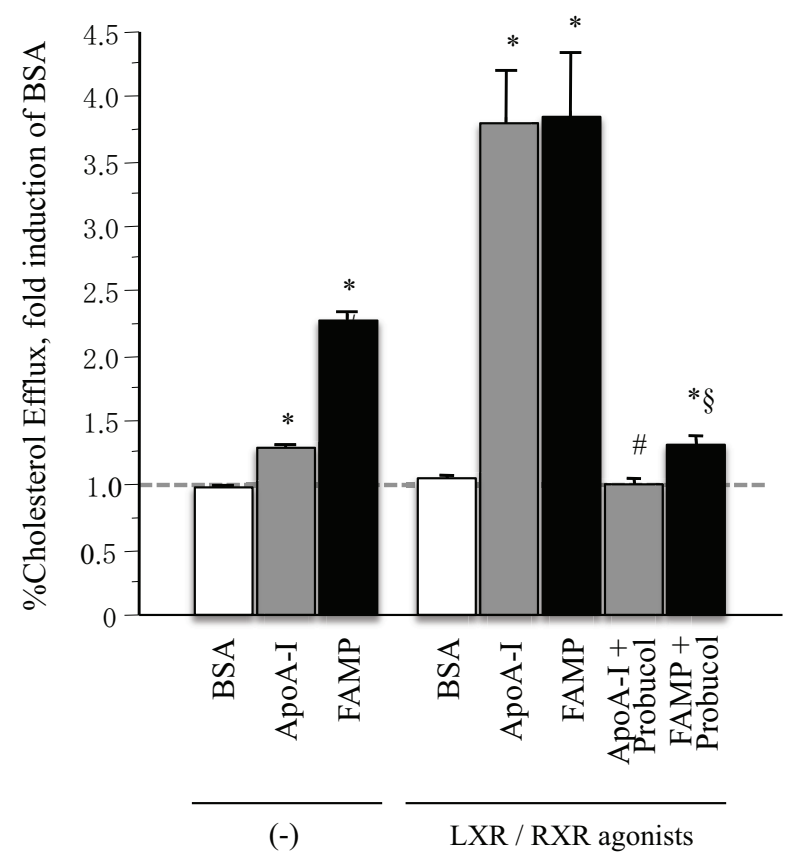

b

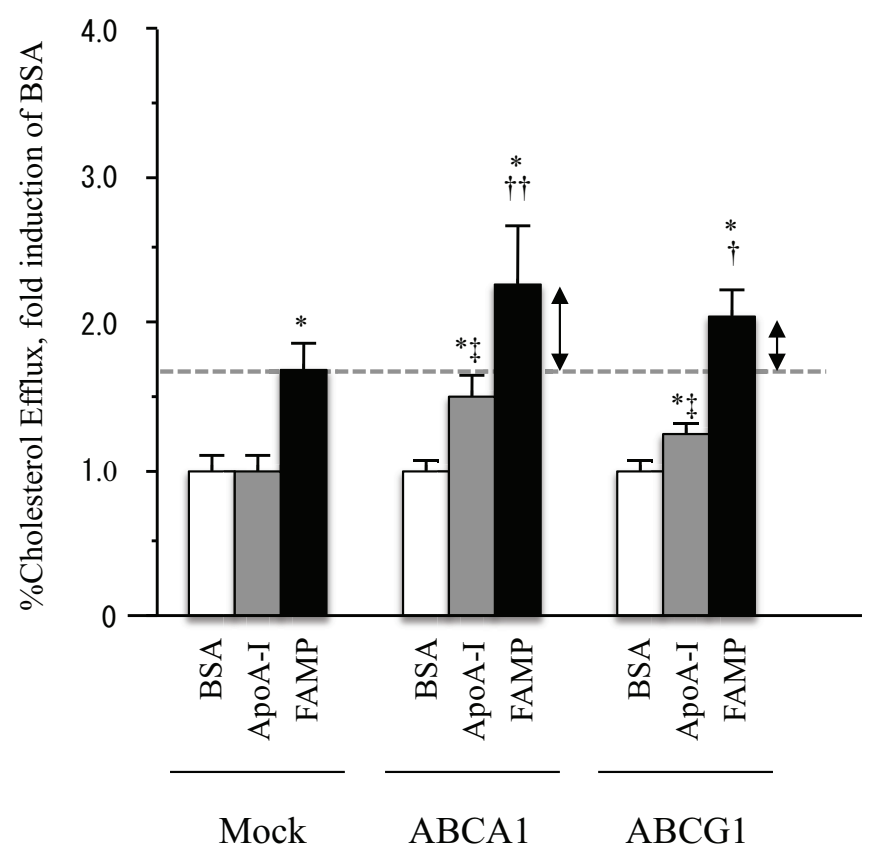

Fig. 2. Effects of FAMP on cellular cholesterol efflux.

(a) ApoA-I- and FAMP5-mediated cholesterol effluxes were measured in cultivated cells in the presence or absence of LXR/RXR agonists and probucol, an ABCA1 specific inhibitor. (b) Cells were transiently transfected with the empty-vector (mock) or with human $A B C A 1$ and $A B C G 1$ cDNAs, and cholesterol efflux was measured after incubation with apoA-I, FAMP, or HDL. ${ }^{*}, P<00.01$ vs. BSA; ${ }^{*}, P<00.01$ vs. apoA-I; ${ }^{\S}, P<00.01$ vs. FAMP; ${ }^{\dagger}, P<00.05$ vs. FAMP in mock; ${ }^{\dagger \dagger}, P<0.01$ vs. FAMP in mock; ${ }^{*}, P<00.01$ vs. apoA-I in mock. Graphs modified from the paper by Uehara et al. ${ }^{38)}$.

(FAMP) is an ApoA-I mimetic peptide developed by our group. First, we synthesized various novel functional ApoA-I mimetic peptides consisting of 24-25 amino acids. In particular, the type 5 FAMP retains a strong amphipathic helical structure and elicits cholesterol efflux through the ABCA1 specific pathway. FAMP may play a physiological role similar to that of human ApoA-I, with its efflux function mediated by ABCA1, because an absence of functional ABCA1 transporters blocked FAMP-stimulated cholesterol efflux ${ }^{33)}$. (Fig. 2a) In contrast to the FAMP-mediated cholesterol effluxes of human full-length ApoA-I, they are not completely abolished under ABCA1-inactivated conditions, such as in cells treated with probucol, an ABCA1 antagonist, and in Tangier macrophages. These results suggested that FAMP functioned in removing cholesterol through the ABCA1 pathway and another specific pathway dependent on ABCG1 transporters that is a potent atheroprotective key molecule in an initial step of HDL metabolism as well as ABCA $1^{34-37)}$. Accordingly, cells transiently transfected with the $A B C A 1$ and $A B C G 1$ genes showed signifi- cantly increased FAMP-mediated effluxes compared with those in the control. (Fig. 2b)

The most important role of ApoA-I is expected to be HDL construction, which depends on its amphipathic properties. FAMP consists of a region of amino acids spanning positions 196-219 of the human ApoA-I, forming a typical alpha-helical conformation and has an amphipathic helix potential in the solution despite being only 24 amino acids long.

FAMP has at least two possible distinct roles in HDL metabolism, particularly pre- $\beta$ HDL production. First, FAMP enhances the cholesterol efflux mediated by ABCA1-dependent and -independent mechanisms, resulting in the generation of nascent pre- $\beta$ HDL particles. In addition to HDL neogenesis, incubation of FAMP with human HDL or plasma generates small HDL particles and charged ApoA-I-rich particles, which migrate as pre- $\beta$ HDL on agarose gel electrophoresis. These observations suggest that incubation of human plasma with FAMP transforms $\alpha$-HDL to pre- $\beta$ HDL and increases HDL function in vitro. (Fig. 3) 


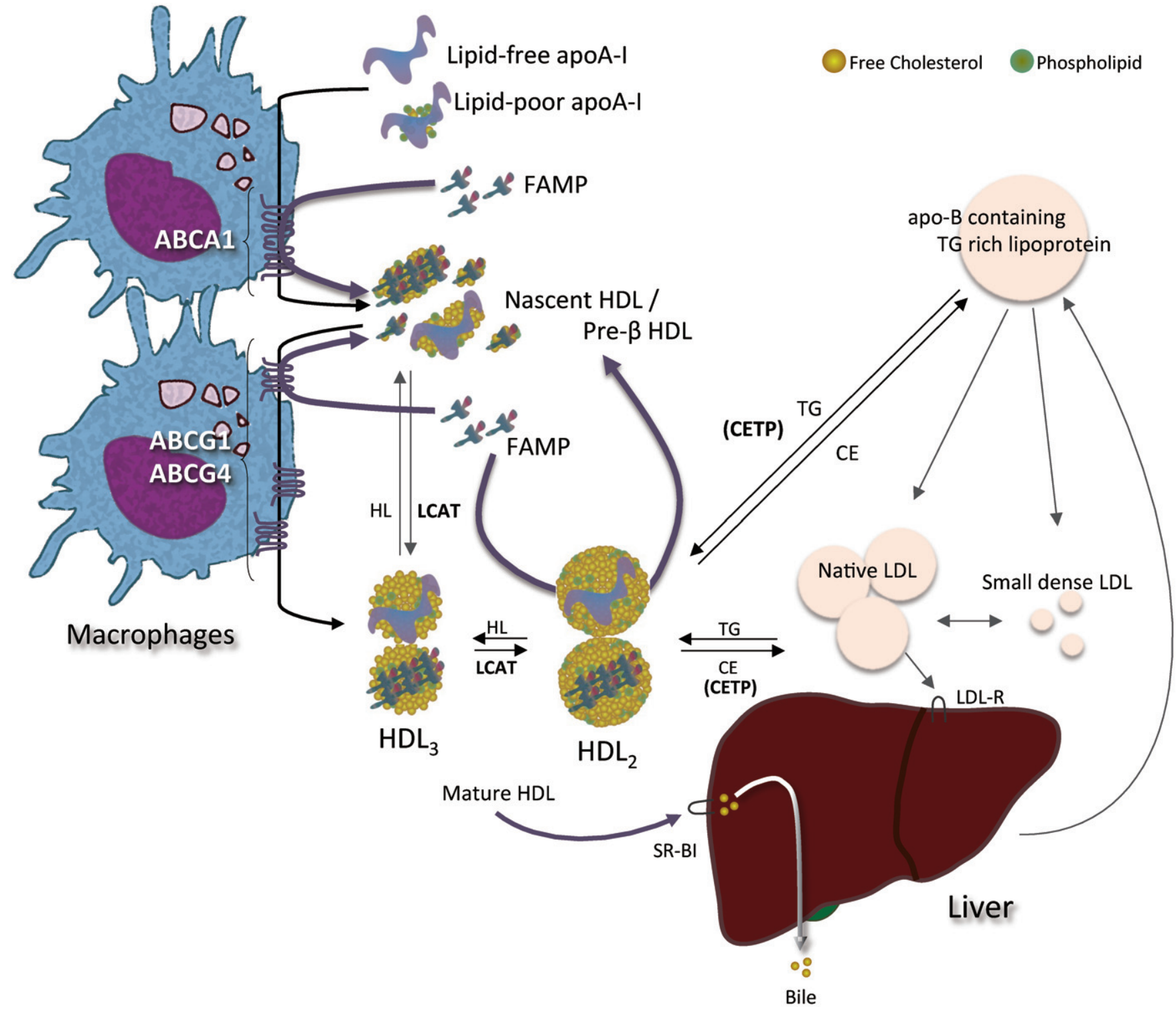

Fig. 3. Illustration of HDL metabolism and suggested function of FAMP in HDL metabolism.

HDL, high-density lipoprotein; LDL, low-density lipoprotein; ABC, ATP-binding cassette transporter; TG, triglyceride; CE, cholesteryl ester; apo, apolipoprotein; CETP, cholesteryl ester transfer protein; LCAT, lecithin cholesterol acyltransferase; HL, hepatic lipase; LDL-R, LDL receptor; SR-BI, scavenger receptor class B type I.

One of the differences between FAMP and $4 \mathrm{~F}$ is modifications of its terminal ends. $4 \mathrm{~F}$ is acetylated at its $\mathrm{N}$ terminus and amidated at its $\mathrm{C}$ terminus, whereas the peptide of the same amino acid sequence with natural amidation at the $\mathrm{N}$ terminus and carboxylation at the $\mathrm{C}$ terminus lacks any function for cholesterol efflux; in contrast, FAMP had no influence because of its terminal end modifications, and these characteristics may enable its functioning in an E. coli or plant expression system.

Treatment with FAMP three times per week for
16 weeks has resulted in a marked inhibition of atherosclerotic plaque progression, whereas plasma HDL cholesterol was not increased in ApoE-deficient mice fed a high-fat $\operatorname{diet}^{38)}$. (Fig. 4) These results strongly suggest that the treatment with FAMP suppresses the atherosclerotic plaque formations by enhancement of the biological HDL function without increasing of HDL cholesterol level. Lipoprotein profiling by HPLC showed that free cholesterol of small HDL particles and cholesterol efflux capacity were significantly improved, according to the results of an analysis of $e x$ 
vivo HDL efflux capacity that is strongly predictive of coronary heart disease, ${ }^{39)}$ and plasma C-reactive protein was reduced by treatment with FAMP. Furthermore, FAMP promotes ABCA1-dependent HDL efflux ex vivo, HDL turnover in vivo, and reverse cholesterol transport in macrophages of transgenic mice that express human CETP, despite decreased plasma HDL cholesterol levels ${ }^{40)}$. FAMP improved HDL functions by increasing pre- $\beta$ HDL and, thus, improved anti-inflammatory and anti-atherogenic functions in vivo. Thus, FAMP is a promising peptide for the suppression of arterial plaque formation in humans.

Besides its therapeutic potential, FAMP exhibits interesting properties that can be applied to the analysis of atherosclerotic plaque. An imaging technique for atherosclerosis applying the physiological HDL function is developed. ApoA-I or its mimetics must penetrate atherosclerotic plaques to remove cholesterol, and this characteristic may be exploited to detect activated arterial plaques. FAMP serves as a unique tracer for positron electron emission tomography (PET). When FAMP is functionalized using the chelator, 1,4,7,10-tetraazacyclododecane-1,4,7,10-tetraacetic acid and labeled with ${ }^{68} \mathrm{Ga}\left({ }^{68} \mathrm{Ga}-\right.$ DOTA-FAMP), it can be used to specifically image atherosclerotic plaques. Thus, atherosclerotic plaques incorporate ${ }^{68} \mathrm{Ga}-$ DOTA-FAMP at a high rate, generating impressive PET images of an aortic plaque in vivo. ${ }^{41)}$

Several fundamental experiments have shown that the administration of ApoA-I or artificial HDL is a novel therapeutic strategy for the treatment of atherosclerosis. CSL-111 is a reconstituted-HDL comprising ApoA-I isolated from human plasma and phosphatidylcholine derived from soybean. A clinical trial pertaining to ACS patients showed that shortterm infusions of CSL-111 or placebo resulted in differences in the coronary atheroma volume; however, the difference between these groups was not statistically significant. ${ }^{42)}$ However, CSL-111 may potentially have beneficial effects such as a significant reduction of $3.4 \%\left(5.34 \mathrm{~mm}^{3}\right)$ in the atheroma volume compared with the baseline value. As observed in the clinical trial of CER-001, clinical trials focus on ACS patients and at times fail to provide the desired outcome. A factor contributing to this failure may be including ACS patients in the study sample. Generally, a positive outcome in clinical trials is more likely to be observed in ACS patients than in other patients such as those with stable angina. Conversely, the biological effects of ACS differ from those of atherosclerosis, specifically ACS results in acute, intensive, and local vascular inflammation; thus, its treatment may require a powerful anti-inflammatory agent as opposed
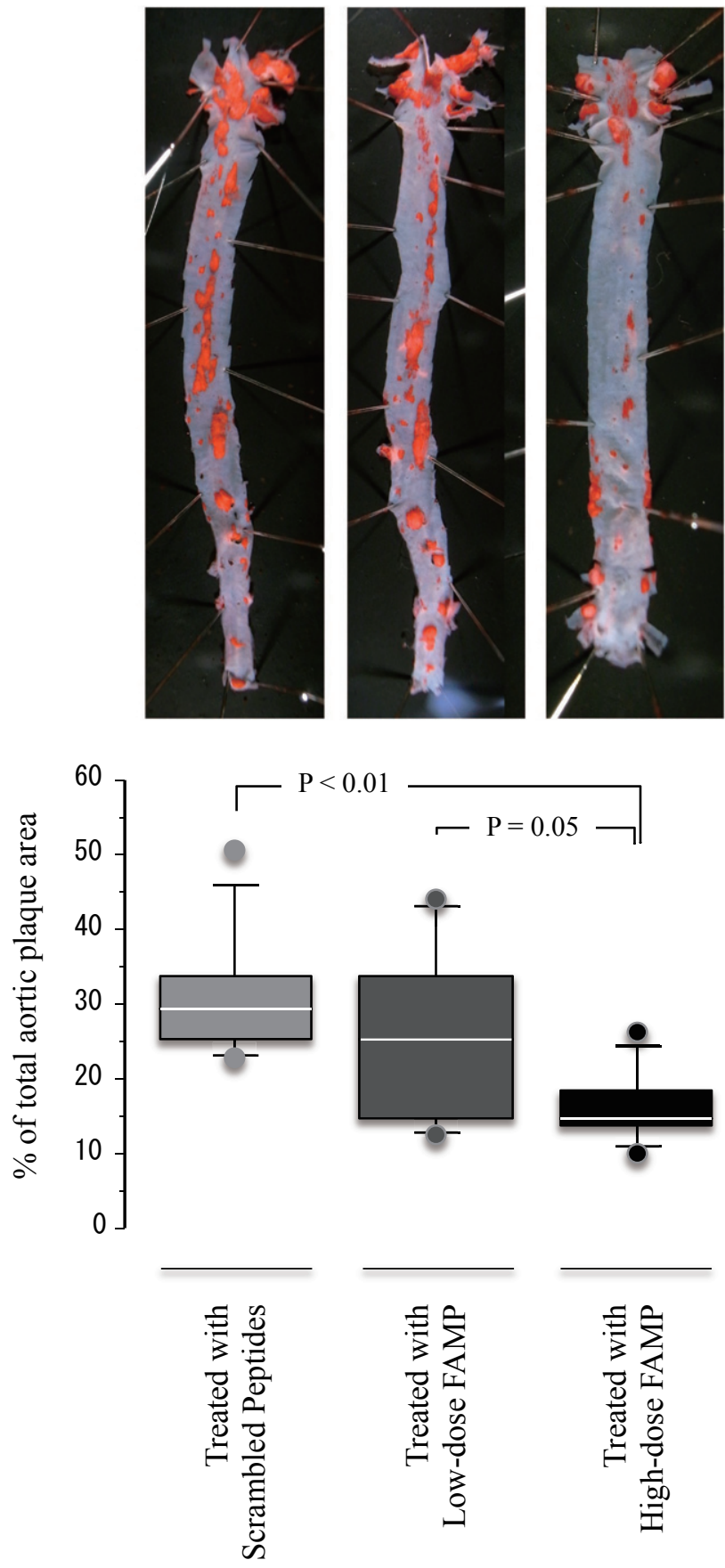

Fig.4. Effect of ApoA-I mimetic peptide, FAMP on atherosclerotic plaque formation in $\mathrm{ApoE}^{-/-}$mice.

$\mathrm{ApoE}^{-/-}$mice fed a high-fat diet were intraperitoneally treated 3 times per week with scrambled FAMP, low-dose FAMP $(10 \mathrm{mg} / \mathrm{kg}$ body weight), or high-dose FAMP ( $50 \mathrm{mg} / \mathrm{kg}$ body weight). Graph and pictures modified from the paper by Uehara et al. ${ }^{38)}$.

to a cholesterol removal agent. Therefore, there is a requirement for further clinical trials to demonstrate the beneficial effects of HDL mimetics. 
In 2014, Rohatgi et al. reported that the HDL cholesterol efflux capacity was inversely correlated with the incidence of cardiovascular events in a population-based cohort ${ }^{43)}$. Their study investigated cholesterol efflux capacity at baseline in 2,924 adults who were free of cardiovascular diseases, and a primary endpoint of the study was cardiovascular event, defined as the first non-fatal myocardial infarction, non-fatal stroke, or coronary revascularization or death from cardiovascular causes. Therefore, in a fully adjusted model of conventional risk factors, HDL cholesterol level, and HDL particle concentration, the cardiovascular risk was reduced by $67 \%$ in the group with a high HDL cholesterol efflux capacity. In a recent clinical trial, the cholesterol efflux capacity was associated with CAD, supporting a therapeutic strategy for improving this capacity and HDL function using HDL mimetics. However, HDL-targeted therapies should continue to be considered as therapeutic options, comprehensively considering the effect of not only the quantity of HDL cholesterol but also the quality of HDL cholesterol.

\section{Conclusion}

Currently, statin treatment is the standard therapy for cardiovascular diseases; this treatment reduces plasma LDL cholesterol levels. However, more than half of the patients have not been able to receive the beneficial effects of this treatment. Thus, targeting HDL may have prospects for treating patients with atherosclerosis. In particular, HDL mimetics, including ApoA-I mimetic peptides such as FAMP have significant atheroprotective potential and may prove to be a therapeutic tool for atherosclerosis.

\section{Acknowledgments}

This work was supported by Grants-in-Aid for Scientific Research (No. 19590874, No. 25461141 and No. 21590960) from the Ministry of Education, Culture, Sports, Science, and Technology of Japan to Dr. Yoshinari Uehara and Dr. Keijiro Saku; and in part by funds from the Central Research Institute of Fukuoka University (2013-2015) to Dr. Yoshinari Uehara and Dr. Keijiro Saku, the Fukuoka University One-Campus Project (2009, 2010, supported in part by the Ministry of Education, Science and Culture of Japan), supported in part by funds (No. 157009) from the Central Research Institute of Fukuoka University to Dr. Yoshinari Uehara and NPO Clinical and Applied Science, Fukuoka, Japan.

\section{Disclosures}

Research and education grants, consulting, and promotional speaking (KS) from Otsuka Co., Asteras, Kowa, Behlinger, Pfizer Co. Ltd, Dainihon Sumitomo Co., SANOFI, Takeda Pharmaceutical Co., Ltd., Bayer, Eli Lilly Co., and TOA EIYO Co., and (KS ,YU) from MSD Co., and Daiichi-sakyo Co. Ltd (clinical research grant). KS is Directors of NPO Clinical and Applied Science, Fukuoka, Japan. KS had a grant from the Public Interest Incorporated Foundation of "Clinical Research Promotion Foundation" in Fukuoka, Japan, and the part of work was transferred into NPO Clinical and Applied Science, Fukuoka, Japan. KS has Endowed Department of Molecular Cardiovascular Therapeutics (YU), Fukuoka University, supported by MSD Co., Ltd., Tokyo, Japan, and Endowed Department of Community and Emergency Medicine, Fukuoka University, supported by Izumi City, Kagoshima, Japan.

\section{References}

1) von Eckardstein A, Nofer JR and Assmann G: High density lipoproteins and arteriosclerosis. Role of cholesterol efflux and reverse cholesterol transport. Arterioscler Thromb Vasc Biol, 2001; 21: 13-27

2) Gordon T, Castelli WP, Hjortland MC, Kannel WB and Dawber TR: High density lipoprotein as a protective factor against coronary heart disease. The Framingham Study. Am J Med, 1977; 62: 707-714

3) Miyamoto-Sasaki M, Yasuda T, Monguchi T, Nakajima $\mathrm{H}$, Mori K, Toh R, Ishida T and Hirata K: Pitavastatin increases HDL particles functionally preserved with cholesterol efflux capacity and antioxidative actions in dyslipidemic patients. J Atheroscler Thromb, 2013; 20: 708716

4) Guyton JR, Slee AE, Anderson T, Fleg JL, Goldberg RB, Kashyap ML, Marcovina SM, Nash SD, O'Brien KD, Weintraub WS, Xu P, Zhao XQ and Boden WE: Relationship of lipoproteins to cardiovascular events: the AIM-HIGH Trial (Atherothrombosis Intervention in Metabolic Syndrome With Low HDL/High Triglycerides and Impact on Global Health Outcomes). J Am Coll Cardiol, 2013; 62: 1580-1584

5) Schwartz GG, Olsson AG, Abt M, Ballantyne CM, Barter PJ, Brumm J, Chaitman BR, Holme IM, Kallend D, Leiter LA, Leitersdorf E, McMurray JJ, Mundl H, Nicholls SJ, Shah PK, Tardif JC, Wright RS and dal-OUTCOMES Investigators.: Effects of dalcetrapib in patients with a recent acute coronary syndrome. N Engl J Med, 2012; 367: 2089-2099

6) Barter PJ, Brewer HB, Jr., Chapman MJ, Hennekens CH, Rader DJ and Tall AR: Cholesteryl ester transfer protein: a novel target for raising HDL and inhibiting atherosclerosis. Arterioscler Thromb Vasc Biol, 2003; 23: 160-167

7) Nicholls SJ, Tuzcu EM, Brennan DM, Tardif JC and Nis- 
sen SE: Cholesteryl ester transfer protein inhibition, highdensity lipoprotein raising, and progression of coronary atherosclerosis: insights from ILLUSTRATE (Investigation of Lipid Level Management Using Coronary Ultrasound to Assess Reduction of Atherosclerosis by CETP Inhibition and HDL Elevation). Circulation, 2008; 118: 2506-2514

8) Voight BF, Peloso GM, Orho-Melander M, FrikkeSchmidt R, Barbalic M, Jensen MK, Hindy G, Holm H, Ding EL, Johnson T, Schunkert H, Samani NJ, Clarke R, Hopewell JC, Thompson JF, Li M, Thorleifsson G, Newton-Cheh C, Musunuru K, Pirruccello JP, Saleheen D, Chen L, Stewart A, Schillert A, Thorsteinsdottir U, Thorgeirsson G, Anand S, Engert JC, Morgan T, Spertus J, Stoll M, Berger K, Martinelli N, Girelli D, McKeown PP, Patterson CC, Epstein SE, Devaney J, Burnett MS, Mooser V, Ripatti S, Surakka I, Nieminen MS, Sinisalo J, Lokki ML, Perola M, Havulinna A, de Faire U, Gigante B, Ingelsson E, Zeller T, Wild P, de Bakker PI, Klungel $\mathrm{OH}$, Maitland-van der Zee AH, Peters BJ, de Boer A, Grobbee DE, Kamphuisen PW, Deneer VH, Elbers CC, Onland-Moret NC, Hofker MH, Wijmenga C, Verschuren WM, Boer JM, van der Schouw YT, Rasheed A, Frossard P, Demissie S, Willer C, Do R, Ordovas JM, Abecasis GR, Boehnke M, Mohlke KL, Daly MJ, Guiducci C, Burtt NP, Surti A, Gonzalez E, Purcell S, Gabriel $S$, Marrugat J, Peden J, Erdmann J, Diemert P, Willenborg C, Konig IR, Fischer M, Hengstenberg C, Ziegler A, Buysschaert I, Lambrechts D, Van de Werf F, Fox KA, El Mokhtari NE, Rubin D, Schrezenmeir J, Schreiber S, Schafer A, Danesh J, Blankenberg S, Roberts R, McPherson R, Watkins H, Hall AS, Overvad K, Rimm E, Boerwinkle E, Tybjaerg-Hansen A, Cupples LA, Reilly MP, Melander O, Mannucci PM, Ardissino D, Siscovick D, Elosua R, Stefansson K, O'Donnell CJ, Salomaa V, Rader DJ, Peltonen L, Schwartz SM, Altshuler D and Kathiresan S: Plasma HDL cholesterol and risk of myocardial infarction: a mendelian randomisation study. Lancet, 2012; 380: 572-580

9) Iwata A, Miura S, Zhang B, Imaizumi S, Uehara Y, Shiomi M and Saku K: Antiatherogenic effects of newly developed apolipoprotein A-I mimetic peptide/phospholipid complexes against aortic plaque burden in Watanabe-heritable hyperlipidemic rabbits. Atherosclerosis, 2011; 218: 300-307

10) Nissen SE, Tsunoda T, Tuzcu EM, Schoenhagen P, Cooper CJ, Yasin M, Eaton GM, Lauer MA, Sheldon WS, Grines CL, Halpern S, Crowe T, Blankenship JC and Kerensky R: Effect of recombinant ApoA-I Milano on coronary atherosclerosis in patients with acute coronary syndromes: a randomized controlled trial. JAMA, 2003; 290: 2292-2300

11) Rubin EM, Krauss RM, Spangler EA, Verstuyft JG and Clift SM: Inhibition of early atherogenesis in transgenic mice by human apolipoprotein AI. Nature, 1991; 353: 265-267

12) Zhang Y, Zanotti I, Reilly MP, Glick JM, Rothblat GH and Rader DJ: Overexpression of apolipoprotein A-I promotes reverse transport of cholesterol from macrophages to feces in vivo. Circulation, 2003; 108: 661-663
13) Mora S, Glynn RJ and Ridker PM: High-density lipoprotein cholesterol, size, particle number, and residual vascular risk after potent statin therapy. Circulation, 2013; 128: 1189-1197

14) Sung KC, Ryu S, Wild SH and Byrne CD: An increased high-density lipoprotein cholesterol/apolipoprotein A-I ratio is associated with increased cardiovascular and allcause mortality. Heart, 2015; 101: 553-558

15) Despres JP and Marette A: Relation of components of insulin resistance syndrome to coronary disease risk. Curr Opin Lipidol, 1994; 5: 274-289

16) Zhang B, Kawachi E, Miura S, Uehara Y, Matsunaga A, Kuroki M and Saku K: Therapeutic approaches to the regulation of metabolism of high-density lipoprotein. Novel HDL-directed pharmacological intervention and exercise. Circ J, 2013; 77: 2651-2663

17) Ornish D, Scherwitz LW, Billings JH, Brown SE, Gould KL, Merritt TA, Sparler S, Armstrong WT, Ports TA, Kirkeeide RL, Hogeboom C and Brand RJ: Intensive lifestyle changes for reversal of coronary heart disease. JAMA, 1998; 280: 2001-2007

18) Michishita R, Tanaka H, Kumahara H, Ayabe M, Tobina T, Yoshimura E, Matsuda T, Higaki Y and Kiyonaga A: Effects of lifestyle modifications on improvement in the blood lipid profiles in patients with dyslipidemia. J Metabolic Synd, 2014; 3, DOI: 10.4172/2167-0943.1000150

19) Weisgraber KH, Bersot TP, Mahley RW, Franceschini G and Sirtori CR: A-I milano apoprotein. Isolation and characterization of a cysteine-containing variant of the A-I apoprotein from human high density lipoproteins. J Clin Invest, 1980; 66: 901-907

20) Gualandri V, Franceschini G, Sirtori CR, Gianfranceschi G, Orsini GB, Cerrone A and Menotti A: AI Milano apoprotein identification of the complete kindred and evidence of a dominant genetic transmission. Am J Hum Genet, 1985; 37: 1083-1097

21) Favari E, Gomaraschi M, Zanotti I, Bernini F, Lee-Rueckert M, Kovanen PT, Sirtori CR, Franceschini G and Calabresi L: A unique protease-sensitive high density lipoprotein particle containing the apolipoprotein A-I(Milano) dimer effectively promotes ATP-binding Cassette A1-mediated cell cholesterol efflux. J Biol Chem, 2007; 282: 5125-5132

22) Franceschini G, Calabresi L, Chiesa G, Parolini C, Sirtori $\mathrm{CR}$, Canavesi $\mathrm{M}$ and Bernini $\mathrm{F}$ : Increased cholesterol efflux potential of sera from ApoA-IMilano carriers and transgenic mice. Arterioscler Thromb Vasc Biol, 1999; 19: 1257-1262

23) Shah PK, Yano J, Reyes O, Chyu KY, Kaul S, Bisgaier CL, Drake $S$ and Cercek B: High-dose recombinant apolipoprotein A-I(milano) mobilizes tissue cholesterol and rapidly reduces plaque lipid and macrophage content in apolipoprotein E-deficient mice. Potential implications for acute plaque stabilization. Circulation, 2001; 103: 3047-3050

24) Parolini C, Marchesi M, Lorenzon P, Castano M, Balconi E, Miragoli L, Chaabane L, Morisetti A, Lorusso V, Martin BJ, Bisgaier CL, Krause B, Newton RS, Sirtori CR and Chiesa G: Dose-related effects of repeated ETC-216 (recombinant apolipoprotein A-I Milano/1-palmitoyl-2- 
oleoyl phosphatidylcholine complexes) administrations on rabbit lipid-rich soft plaques: in vivo assessment by intravascular ultrasound and magnetic resonance imaging. J Am Coll Cardiol, 2008; 51: 1098-1103

25) Nissen SE, Tuzcu EM, Schoenhagen P, Brown BG, Ganz P, Vogel RA, Crowe T, Howard G, Cooper CJ, Brodie B, Grines CL, DeMaria AN and Investigators R: Effect of intensive compared with moderate lipid-lowering therapy on progression of coronary atherosclerosis: a randomized controlled trial. JAMA, 2004; 291: 1071-1080

26) Tardy C, Goffinet M, Boubekeur N, Ackermann R, Sy G, Bluteau A, Cholez G, Keyserling C, Lalwani N, Paolini JF, Dasseux JL, Barbaras R and Baron R: CER-001, a HDL-mimetic, stimulates the reverse lipid transport and atherosclerosis regression in high cholesterol diet-fed LDL-receptor deficient mice. Atherosclerosis, 2014; 232: 110-118

27) Tardif JC, Ballantyne CM, Barter P, Dasseux JL, Fayad ZA, Guertin MC, Kastelein JJ, Keyserling C, Klepp H, Koenig W, L'Allier PL, Lesperance J, Luscher TF, Paolini JF, Tawakol A, Waters DD and Can HDLISQARI: Effects of the high-density lipoprotein mimetic agent CER-001 on coronary atherosclerosis in patients with acute coronary syndromes: a randomized trial. Eur Heart J, 2014; 35: 3277-3286

28) Van Lenten BJ, Wagner AC, Jung CL, Ruchala P, Waring AJ, Lehrer RI, Watson AD, Hama S, Navab M, Anantharamaiah GM and Fogelman AM: Anti-inflammatory apoA-I-mimetic peptides bind oxidized lipids with much higher affinity than human apoA-I. J Lipid Res, 2008; 49: 2302-2311

29) Sharma S, Umar S, Potus F, Iorga A, Wong G, Meriwether D, Breuils-Bonnet S, Mai D, Navab K, Ross D, Navab M, Provencher S, Fogelman AM, Bonnet S, Reddy ST and Eghbali M: Apolipoprotein A-I mimetic peptide $4 \mathrm{~F}$ rescues pulmonary hypertension by inducing microRNA-193-3p. Circulation, 2014; 130: 776-785

30) Navab M, Yu R, Gharavi N, Huang W, Ezra N, Lotfizadeh A, Anantharamaiah GM, Alipour N, Van Lenten BJ, Reddy ST and Marelli D: High-density lipoprotein: antioxidant and anti-inflammatory properties. Curr Atheroscler Rep, 2007; 9: 244-248

31) Bloedon LT, Dunbar R, Duffy D, Pinell-Salles P, Norris R, DeGroot BJ, Movva R, Navab M, Fogelman AM and Rader DJ: Safety, pharmacokinetics, and pharmacodynamics of oral apoA-I mimetic peptide D-4F in high-risk cardiovascular patients. J Lipid Res, 2008; 49: 1344-1352

32) Watson CE, Weissbach N, Kjems L, Ayalasomayajula S, Zhang Y, Chang I, Navab M, Hama S, Hough G, Reddy ST, Soffer D, Rader DJ, Fogelman AM and Schecter A: Treatment of patients with cardiovascular disease with L-4F, an apo-A1 mimetic, did not improve select biomarkers of HDL function. J Lipid Res, 2011; 52: 361373

33) Uehara Y, Ando S, Oniki K, Abe S, Yahiro E, Tanigawa H, Miura SI and Saku K: FAMP, a novel apoA-I mimetic peptide promotes HDL via ABCA1-dependent cholesterol efflux. ATHEROSCLEROSIS SUPP, 2010; 11: 3-3

34) Furuyama S, Uehara Y, Zhang B, Baba Y, Abe S, Iwamoto T, Miura S and Saku K: Genotypic Effect of ABCG1 gene promoter $-257 \mathrm{~T}>\mathrm{G}$ polymorphism on coronary artery disease severity in Japanese men. J Atheroscler Thromb, 2009; 16: 194-200

35) Uehara Y and Saku K: High-density lipoprotein and atherosclerosis: Roles of lipid transporters. World J Cardiol, 2014; 6: 1049-1059

36) Kennedy MA, Barrera GC, Nakamura K, Baldan A, Tarr P, Fishbein MC, Frank J, Francone OL and Edwards PA: ABCG1 has a critical role in mediating cholesterol efflux to HDL and preventing cellular lipid accumulation. Cell Metab, 2005; 1: 121-131

37) Mauldin JP, Srinivasan S, Mulya A, Gebre A, Parks JS, Daugherty A and Hedrick CC: Reduction in ABCG1 in Type 2 diabetic mice increases macrophage foam cell formation. J Biol Chem, 2006; 281: 21216-21224

38) Uehara Y, Ando S, Yahiro E, Oniki K, Ayaori M, Abe S, Kawachi E, Zhang B, Shioi S, Tanigawa H, Imaizumi S, Miura $S$ and Saku K: FAMP, a novel apoA-I mimetic peptide, suppresses aortic plaque formation through promotion of biological HDL function in ApoE-deficient mice. J Am Heart Assoc, 2013; 2: e000048, DOI: 10.1161/ JAHA.113.000048

39) Khera AV, Cuchel M, de la Llera-Moya M, Rodrigues A, Burke MF, Jafri K, French BC, Phillips JA, Mucksavage ML, Wilensky RL, Mohler ER, Rothblat GH and Rader DJ: Cholesterol efflux capacity, high-density lipoprotein function, and atherosclerosis. N Engl J Med, 2011; 364: $127-135$

40) Shimizu T, Tanigawa H, Miura S, Kuwano T, Takata K, Suematsu Y, Imaizumi S, Yahiro E, Zhang B, Uehara Y and Saku K: Newly developed apolipoprotein A-I mimetic peptide promotes macrophage reverse cholesterol transport in vivo. Int J Cardiol, 2015; 192: 82-88

41) Kawachi E, Uehara Y, Hasegawa K, Yahiro E, Ando S, Wada Y, Yano T, Nishikawa H, Shiomi M, Miura S, Watanabe Y and Saku K: Novel molecular imaging of atherosclerosis with gallium-68-labeled apolipoprotein A-I mimetic peptide and positron emission tomography. Circ J, 2013; 77: 1482-1489

42) Tardif JC, Gregoire J, L'Allier PL, Ibrahim R, Lesperance J, Heinonen TM, Kouz S, Berry C, Basser R, Lavoie MA, Guertin MC, Rodes-Cabau J, Effect of rHDL on Atherosclerosis-Safety and Efficacy (ERASE) Investigators.: Effects of reconstituted high-density lipoprotein infusions on coronary atherosclerosis: a randomized controlled trial. JAMA, 2007; 297: 1675-1682

43) Rohatgi A, Khera A, Berry JD, Givens EG, Ayers CR, Wedin KE, Neeland IJ, Yuhanna IS, Rader DR, de Lemos JA and Shaul PW: HDL cholesterol efflux capacity and incident cardiovascular events. N Engl J Med, 2014; 371: 2383-2393 